Lee Peng Yee and Ng Wee Leng, Mathematics Division, School of Science, Nanyang Technological University, Singapore 259756

\title{
THE RADON-NIKODÝM THEOREM FOR THE HENSTOCK INTEGRAL IN EUCLIDEAN SPACE
}

\begin{abstract}
We prove the Radon-Nikodým theorem for the Henstock integral and hence give a complete characterization of the primitive of a Henstock integrable function in Euclidean space.
\end{abstract}

The descriptive definition of the Henstock integral on the real line is wellknown. More precisely, a function $f$ is Henstock integrable on a closed interval $[a, b]$ if and only if there exists a function $F$ which is generalized absolutely continuous in the restricted sense on $[a, b]$ such that $F^{\prime}(x)=f(x)$ almost everywhere. (See [3, Theorem 6.22] or [6].) Thus the primitive of a Henstock integrable function on the real line is totally characterized. So far, no full characterization of the primitive of a Henstock integrable function in Euclidean space has been found. In this note, we shall prove the Radon-Nikodým theorem for the Henstock integral in Euclidean space, and hence characterize the primitive of a Henstock integrable function.

We first define the Henstock integral in Euclidean space. Let $I$ be an interval in $n$-dimensional Euclidean space $\mathbb{R}^{n}$, that is, the set of all points $x=\left(x_{1}, \ldots, x_{n}\right)$ with $a_{j} \leq x_{j} \leq b_{j}$ for $j=1,2, \ldots, n$. We write $[a, b]=$ $\left[a_{1}, b_{1}\right] \times \cdots \times\left[a_{n}, b_{n}\right]$ where $a=\left(a_{1}, \ldots, a_{n}\right)$ and $b=\left(b_{1}, \ldots, b_{n}\right)$ and call $[a, b]$ an interval. If $I=[\alpha, \beta]$ with $\alpha=\left(\alpha_{1}, \ldots, \alpha_{n}\right)$ and $\beta=\left(\beta_{1}, \ldots, \beta_{n}\right)$, any point $\gamma=\left(\gamma_{1}, \ldots, \gamma_{n}\right)$ with $\gamma_{j}=\alpha_{j}$ or $\beta_{j}$ is called a vertex of $I$. Assume that the distance between $x=\left(x_{1}, \ldots, x_{n}\right)$ and $y=\left(y_{1}, \ldots, y_{n}\right)$ is defined to be

$$
\|x-y\|=\left[\sum_{j=1}^{n}\left(x_{j}-y_{j}\right)^{2}\right]^{1 / 2} .
$$

Key Words: Henstock integral, $A C G_{\Delta}$, Radon-Nikodým theorem, (L)-condition, basic condition

Mathematical Reviews subject classification: 26A39

Received by the editors April 15, 1996 
An open sphere $S(x, r)$ with center $x$ and radius $r$ is the set of all $y$ such that $\|y-x\|<r$.

We shall call a finite union of non-overlapping intervals (that is, their interiors are disjoint) an elementary set. Note that intervals are themselves elementary sets. A partial division $D$ of $E$ is a finite collection of interval-point pairs $(I, x)$ with the intervals non-overlapping, and their union a subset of $E$. If a partial division $D$ is such that the union of the intervals is $E$, we call $D$ a division of $E$. We shall write $D=\{(I, x)\}$. As usual, $x$ is called the associated point of $I$.

Let $\mathbb{R}^{+}$denote the set of all positive real numbers and let $\delta: E \rightarrow \mathbb{R}^{+}$ be a positive function. A partial division $D=\{(I, x)\}$ is said to be $\delta$-fine if, for each interval-point pair $(I, x)$, we have $I \subset S(x, \delta(x))$ and where $x$ is a vertex of $I$. Since a division of $E$ is a partial division of $E$, a $\delta$-fine division is similarly defined.

We also call a finite collection $P$ of non-overlapping intervals $I$ whose union is a subset of $E$ a partial partition of $E$ and we write $P=\{I\}$. Again, if a partial partition $P$ is such that the union of the intervals is $E$, we call $P$ a partition of $E$.

The volume of an interval $I=[a, b]$, denoted by $v(I)$, is defined by

$$
v(I)=\prod_{j=1}^{n}\left(b_{j}-a_{j}\right)
$$

We recall that two intervals $I_{1}$ and $I_{2}$ are non-overlapping if $v\left(I_{1} \cap I_{2}\right)=0$.

If a set $X \subset E$ is Lebesgue measurable, then the Lebesgue measure of $X$, denoted by $\nu(X)$, is defined by

$$
\nu(X)=\inf \left\{\sum_{i=1}^{\infty} v\left(I_{i}\right): X \subset \bigcup_{i=1}^{\infty} I_{i}\right\},
$$

where $I_{i}, i=1,2, \ldots$, are intervals in $E$. When $f(x)=g(x)$ for all $x \in E \backslash X$ with $\nu(X)=0$, we say that $f=g$ almost everywhere in $E$.

Definition 1. A real-valued function $f$ defined on $E$ is said to be Henstock integrable on $E$ with integral value $F(E)$ if for every $\varepsilon>0$, there exists $\delta$ : $E \rightarrow \mathbb{R}^{+}$such that for any $\delta$-fine division $D=\{(I, x)\}$ of $E$,

$$
\left|(D) \sum f(x) v(I)-F(E)\right|<\varepsilon .
$$

Here $(D) \sum$ denotes the sum over all interval-point pairs $(I, x)$ in $D$. As usual, we write $(H) \int_{E} f d \nu=F(E)$. 
This integral is uniquely determined and the set of all Henstock integrable functions is closed under addition and scalar multiplication. Furthermore, the integral as an interval function is finitely additive and Henstock's lemma holds. (See [3, Chapter 1].) An interval function $F$ is finitely additive (respectively finitely super-additive) in the sense that if $I=\bigcup_{i=1}^{m} I_{i}$ and the $I_{i}$ are non-overlapping, then

$$
F(I)=\sum_{i=1}^{m} F\left(I_{i}\right) \quad\left(\text { respectively } F(I) \geq \sum_{i=1}^{m} F\left(I_{i}\right)\right) .
$$

Next, we state without proof the Radon-Nikodým theorem for the Lebesgue integral. (See [2, Theorem 19.23].) Throughout the rest of this note, we shall let an elementary set $E$ be fixed.

Theorem 2. (Radon-Nikodým Theorem for the Lebesgue Integral). Let F be a non-negative finitely additive real-valued function defined on the set of all measurable subsets $Y$ of $E$ which is absolutely continuous with respect to the Lebesgue measure $\nu$. Then there exists a non-negative Lebesgue integrable function $f$ on $E$ such that, for any measurable subset $Y$ of $E$,

$$
F(Y)=(L) \int_{Y} f d \nu
$$

where $(L) \int_{Y} f d \nu$ denotes the Lebesgue integral of $f$ over $Y$.

We note that, in the above theorem, $f$ is unique in the sense that if $g$ is any non-negative Lebesgue integrable function for which (1) holds with $f$ replaced by $g$, then $f=g$ almost everywhere in $E$.

Recall that $F$ is absolutely continuous with respect to $\nu$ if and only if, for every $\varepsilon>0$, there exists $\eta>0$ such that, for every measurable subset $Y$ of $E$ satisfying the condition $\nu(Y)<\eta$, we have $|F(Y)|<\varepsilon$, where $|\cdot|$ denotes the measure on the real line. It is known that a function $f$ is Lebesgue integrable on $E$ if and only if it is absolutely Henstock integrable on $E$, i.e. both $f$ and $|f|$ are Henstock integrable on $E$. (See [3, page 110].)

Throughout this note, $F$ will denote a finitely additive real-valued function defined on the set of all elementary subsets of $E$. Our objective is to define a sequence of non-negative finitely additive measures on $E$ in terms of $F$ so that we can apply Theorem 2 to obtain a sequence of Lebesgue integrable functions, and consequently a Henstock integrable function. We start with a few definitions. 
Definition 3. A function $F$ is said to be $A C(E)$ if for every $\varepsilon>0$, there exists $\eta>0$ such that for every partial partition $P=\{I\}$ of $E$ satisfying the condition $(P) \sum \nu(I)<\eta$, we have $(P) \sum|F(I)|<\varepsilon$.

Definition 4. Let $X$ be a subset of $E$. We say that $F$ is $A C_{\Delta}(X)$ if for every $\varepsilon>0$, there exist $\delta: E \rightarrow \mathbb{R}^{+}$and $\eta>0$ such that, for every $\delta$ fine partial division $D=\{(I, x)\}$ of $E$ with $x \in X$ satisfying the condition $(D) \sum \nu(I)<\eta$, we have $\left|(D) \sum F(I)\right|<\varepsilon$. If there exist closed sets $X_{1}$, $X_{2}, \cdots$ whose union is $E$ such that $F$ is $A C_{\Delta}\left(X_{i}\right)$ for each $i$, then we say that $F$ is $A C G_{\Delta}$ on $E$.

Definition 5. A function $F$ is said to be strongly $A C G_{\Delta}$ on $E$ if there exist closed sets $X_{1}, X_{2}, \cdots$ whose union is $E$ such that $F$ is $A C_{\Delta}\left(X_{i}\right)$ for each $i$, and if the following $(L)$-condition on $E$ holds : for every interval $I_{0}$ in $E$ and for every $\varepsilon>0$ there is a positive integer $N$ such that for any $i \geq N$ there exists $\delta_{i}: E \rightarrow \mathbb{R}^{+}$satisfying the condition that for every $\delta_{i}$-fine division $D=\{(I, x)\}$ of $I_{0}$, we have

$$
\left|(D) \sum_{x \notin X_{i}} F(I)\right|<\varepsilon .
$$

Here $(D) \sum_{x \notin X_{i}}$ sums over all interval-point pairs $(I, x)$ in $D$ with $x \notin X_{i}$.

In the above definition, we may assume that $\delta_{i}$ is a candidate for the positive function $\delta$ in the definition of $F$ being $A C_{\Delta}\left(X_{i}\right)$ for each $i$.

We remark that the primitive $F$ of a Henstock integrable function $f$ as an interval function is $A C G_{\Delta}$ on $E$ as is seen in [5]. Furthermore, $F$ satisfies the $(L G)$-condition as defined in [4, Theorem 7]. With the aid of Henstock's Lemma, we see that the $(L)$-condition is equivalent to the $(L G)$-condition. Hence $F$ is strongly $A C G_{\Delta}$.

Now let us begin with an interval function $F$ which is $A C G_{\Delta}$ on $E$. Then there exist closed sets $X_{1}, X_{2}, \cdots$ whose union is $E$ such that for every $i$ and for every $\varepsilon>0$ the condition in the definition of $A C_{\Delta}(X)$ in Definition 4 holds with $X, \delta$ and $\eta$ replaced by $X_{i}, \delta_{i}$ and $\eta_{i}$ respectively. For each $i$ and for each interval $J$ in $E$, we define

$$
G_{i}(J)=\inf _{\delta} \sup _{D_{\delta}}\left(D_{\delta}\right) \sum_{x \in X_{i}} F(I)
$$

and 


$$
\left|G_{i}\right|(J)=\inf _{\delta} \sup _{D_{\delta}}\left(D_{\delta}\right) \sum_{x \in X_{i}}|F(I)|
$$

where in each case above the infimum is over all positive functions $\delta$ and the supremum is over all $\delta$-fine divisions $D_{\delta}=\{(I, x)\}$ of $J$. We proceed to define, for each interval $J$ in $E$,

$$
F_{i}(J)=\inf _{P}(P) \sum G_{i}(I)
$$

and

$$
\left|F_{i}\right|(J)=\inf _{P}(P) \sum\left|G_{i}\right|(I),
$$

where in each case the infimum is over all partitions $P=\{I\}$ of $J$.

Throughout the remainder of this note, we will call the sequence $\left\{F_{i}\right\}$ of interval functions, where each $F_{i}$ is defined as in (2), the derived sequence of F.

We shall prove that $G_{i}$ and $\left|G_{i}\right|$ are finitely super-additive functions over non-overlapping intervals, whereas $F_{i}$ and $\left|F_{i}\right|$ are finitely additive. Let $J=$ $J_{1} \cup J_{2}$ where $J_{1}$ and $J_{2}$ are non-overlapping intervals in $E$. By the definition of $G_{i}$, for every $\delta(x)>0$, there exist $\delta$-fine divisions $D_{1}$ and $D_{2}$, of $J_{1}$ and $J_{2}$ respectively, such that

$$
\begin{aligned}
G_{i}\left(J_{1}\right)+G_{i}\left(J_{2}\right) & \leq\left(D_{1}\right) \sum_{x \in X_{i}} F(I)+\left(D_{2}\right) \sum_{x \in X_{i}} F(I)+2 \varepsilon \\
& =\left(D_{1} \cup D_{2}\right) \sum F(I)+2 \varepsilon,
\end{aligned}
$$

where $D_{1} \cup D_{2}$ is a $\delta$-fine division of $J_{1} \cup J_{2}$. It follows that

$$
G_{i}\left(J_{1}\right)+G_{i}\left(J_{2}\right) \leq G_{i}(J)+2 \varepsilon .
$$

Since $\varepsilon$ is arbitrary, we have proved that $G_{i}$ is finitely super-additive. The fact that $F_{i}$ is finitely additive is standard as shown in [3]. Similarly, we can show that $\left|G_{i}\right|$ is finitely super-additive whereas $\left|F_{i}\right|$ is finitely additive over non-overlapping intervals.

Since $F$ is $A C G_{\Delta}$ on $E$ and $A C_{\Delta}\left(X_{i}\right)$ for each $i$, the functions $G_{i}$ and $\left|G_{i}\right|$ are well-defined and are $A C(E)$ for each $i$. It follows that $F_{i}$ and $\left|F_{i}\right|$ are also $A C(E)$ for each $i$.

For each interval $I$, we further define

$$
F_{i}^{+}(I)=\frac{\left|F_{i}\right|(I)+F_{i}(I)}{2}
$$


and

$$
F_{i}^{-}(I)=\frac{\left|F_{i}\right|(I)-F_{i}(I)}{2} .
$$

It is easy to see that $F_{i}^{+}$and $F_{i}^{-}$are well-defined non-negative interval functions such that, for each interval $I$,

$$
F_{i}(I)=F_{i}^{+}(I)-F_{i}^{-}(I)
$$

Since $F_{i}$ and $\left|F_{i}\right|$ are finitely additive over non-overlapping intervals and are $A C(E)$ for each $i$, so are $F_{i}^{+}$and $F_{i}^{-}$.

Let us extend the domains of $F_{i}^{+}$and $F_{i}^{-}$to all measurable sets. For each measurable subset $X$ of $E$, we define

$$
F_{i}^{++}(X)=\inf \left\{\sum_{j=1}^{\infty} F_{i}^{+}\left(I_{j}\right): X \subset \bigcup_{j=1}^{\infty} I_{j}\right\}
$$

and

$$
F_{i}^{--}(X)=\inf \left\{\sum_{j=1}^{\infty} F_{i}^{-}\left(I_{j}\right): X \subset \bigcup_{j=1}^{\infty} I_{j}\right\},
$$

where the $I_{j}, j=1,2, \ldots$ are intervals in $E$. Since $F_{i}^{+}$and $F_{i}^{-}$are $A C(E)$, the functions $F_{i}^{++}$and $F_{i}^{--}$agree with $F_{i}^{+}$and $F_{i}^{-}$, respectively, on each interval in $E$ and are additive over disjoint measurable sets.

We shall prove the following lemmas.

Lemma 6. The functions $F_{i}^{++}$and $F_{i}^{--}$are absolutely continuous with respect to the Lebesgue measure $\nu$.

Proof. Let $\varepsilon>0$ be given and let $\eta>0$ be as in the definition of $F_{i}^{+}$being $A C(E)$. Suppose $X$ is a measurable set such that $\nu(X)<\eta$ and let $\left\{I_{k}\right\}_{k=1}^{\infty}$ be a sequence of non-overlapping intervals in $E$ whose union contains $X$ and is such that $\sum_{k=1}^{\infty} \nu\left(I_{k}\right)<\eta$. Then

$$
F_{i}^{++}(X) \leq \sum_{k=1}^{\infty} F_{i}^{+}\left(I_{k}\right)<\varepsilon
$$

It follows similarly that $F_{i}^{--}$is also absolutely continuous with respect to $\nu$. 
Lemma 7. Let $I_{0}$ be an interval in $E$. Suppose $F$ is strongly $A C G_{\Delta}$ on $E$ and $F_{i}$ is as defined in (2). Then $F_{i}\left(I_{0}\right) \rightarrow F\left(I_{0}\right)$ as $i \rightarrow \infty$.

Proof. Let $\varepsilon>0$ be given and let the sequence of closed sets $X_{1}, X_{2}, \cdots$, the sequence of positive functions $\delta_{1}, \delta_{2}, \cdots$ as well as the positive integer $N$ be as in Definition 5. Let $i \geq N$ be fixed. We first choose a partition $P_{0}=\left\{J_{1}, \ldots, J_{m}\right\}$ of $I_{0}$ such that

$$
F_{i}\left(I_{0}\right)-\frac{\varepsilon}{2}<\sum_{k=1}^{m} G_{i}\left(J_{k}\right)<F_{i}\left(I_{0}\right)+\frac{\varepsilon}{2} .
$$

For each $k=1,2, \ldots, m$, there exists a $\delta_{i}$-fine division $D_{k}$ of $J_{k}$ such that

$$
G_{i}\left(J_{k}\right)-\frac{\varepsilon}{2 m}<\left(D_{k}\right) \sum_{x \in X_{i}} F(I)<G_{i}\left(J_{k}\right)+\frac{\varepsilon}{2 m} \text {. }
$$

We then define $D_{0}=\bigcup_{k=1}^{m} D_{k}$. Clearly $D_{0}$ is a $\delta_{i}$-fine division of $I_{0}$ and

$$
F_{i}\left(I_{0}\right)-\varepsilon \leq\left(D_{0}\right) \sum_{x \in X_{i}} F(I)<F_{i}\left(I_{0}\right)+\varepsilon
$$

where $\left(D_{0}\right) \sum_{x \in X_{i}} F(I)=F\left(I_{0}\right)-\left(D_{0}\right) \sum_{x \notin X_{i}} F(I)$ in view of the additivity of $F$. It follows that

$$
\left|F\left(I_{0}\right)-F_{i}\left(I_{0}\right)-\left(D_{0}\right) \sum_{x \notin X_{i}} F(I)\right|<\varepsilon .
$$

By virtue of the $(L)$-condition, we have

$$
\left|\left(D_{0}\right) \sum_{x \notin X_{i}} F(I)\right|<\varepsilon .
$$

Consequently, $\left|F_{i}\left(I_{0}\right)-F\left(I_{0}\right)\right|<2 \varepsilon$ and the result follows.

Definition 8. A sequence $\left\{F_{i}\right\}$ of interval functions is said to satisfy the basic condition with an interval function $F$ if for every $\varepsilon>0$ there is a function $M(x)$ taking integer values such that for infinitely many $m(x) \geq M(x)$ there is $\delta: E \rightarrow \mathbb{R}^{+}$satisfying the condition that for any $\delta$-fine division $D=\{(I, x)\}$ of $E$,

$$
\left|(D) \sum F_{m(x)}(I)-F(E)\right|<\varepsilon .
$$


What follows is a result which we will need later.

Theorem 9. (Lee, [3, Theorem 21.4].) Let the functions $f_{i}, i=1,2, \ldots$ be Henstock integrable on $E$ with primitives $F_{i}, i=1,2, \ldots$ Suppose $f_{i}(x) \rightarrow$ $f(x)$ almost everywhere in $E$ as $i \rightarrow \infty$, and $F_{i}(I)$ converges to $F(I)$ for every interval $I \subset E$. Then in order that $f$ should be Henstock integrable on $E$ with primitive $F$, it is necessary and sufficient that the sequence $\left\{F_{i}\right\}$ of primitives satisfies the basic condition with $F$.

We are now ready to prove the Radon-Nikodým Theorem for the Henstock integral which is stated in the following theorem.

Theorem 10. Let $F$ be a finitely additive function strongly $A C G_{\Delta}$ on $E$ such that the derived sequence of $F$ satisfies the basic condition with $F$. Then there exists a Henstock integrable function $f$ defined on $E$ such that

$$
F\left(E_{0}\right)=(H) \int_{E_{0}} f d \nu
$$

for all elementary subsets $E_{0}$ of $E$. Moreover, $f$ is unique in the sense that, if $g$ is any Henstock integrable function for which (6) holds with $f$ replaced by $g$, then $f=g$ almost everywhere in $E$.

Proof. Let $\varepsilon>0$ be given and let $X_{1}, X_{2}, \cdots$ and $N$ be as in Definition 5 . We may assume that $X_{i} \subset X_{i+1}$ for $i=1,2, \ldots$ We define $F_{i},\left|F_{i}\right|$ and subsequently $F_{i}^{++}$and $F_{i}^{--}$as in (2), (3), (4) and (5) respectively. We recall that $F_{i}^{++}$and $F_{i}^{--}$are non-negative functions finitely additive over disjoint measurable sets. Further, by Lemma 6 , the functions $F_{i}^{++}$and $F_{i}^{--}$are absolutely continuous with respect to $\nu$. Hence, by Theorem 2, for each $i$ there exist non-negative Lebesgue integrable functions $f_{i}^{+}$and $f_{i}^{-}$on $E$ such that

$$
F_{i}^{++}(X)=(L) \int_{X} f_{i}^{+} d \nu \text { and } F_{i}^{--}(X)=(L) \int_{X} f_{i}^{-} d \nu
$$

for all measurable subsets $X$ of $E$. It follows from the definitions of $G_{i}$ and $\left|G_{i}\right|$ that whenever $I \cap X_{i}=\emptyset$, we have $G_{i}(I)=0$ and $\left|G_{i}\right|(I)=0$. Consequently, $F_{i}^{++}(I)=0$ and $F_{i}^{--}(I)=0$ whenever $I \cap X_{i}=\emptyset$. That is,

$$
\text { (L) } \int_{I} f_{i}^{+} d \nu=0 \text { and }(L) \int_{I} f_{i}^{-} d \nu=0
$$

for all intervals $I$ such that $I \cap X_{i}=\emptyset$. Therefore $f_{i}^{+}$and $f_{i}^{-}$vanish almost everywhere in $E \backslash X_{i}$. Now for each $i=1,2, \ldots$, we define 


$$
f_{i}(x)=f_{i}^{+}(x)-f_{i}^{-}(x)
$$

for each $x \in E$ and define the function $f$ on $E$ given by $f(x)=f_{i}(x)$ for $x \in X_{i}, i=1,2, \ldots$. The function $f$ is well-defined due to the uniqueness of $f_{i}$ on $X_{i}$ and obviously $f_{i}(x) \rightarrow f(x)$ almost everywhere in $E$ as $i \rightarrow \infty$. Also note that $f_{i}$ is Lebesgue integrable on $E$ and so is Henstock integrable on $E$ with primitive $F_{i}$ for each $i$. Furthermore, $F_{i}(I)$ converges to $F(I)$ as $i \rightarrow \infty$ for every interval $I \subset E$ by virtue of Lemma 7 . So since the derived sequence of $F$ also satisfies the basic condition with $F$, by Theorem 9 , the function $f$ is Henstock integrable on $E$ with primitive $F$. Now, for each interval $I$ in $E$,

$$
F_{i}(I)=F_{i}^{++}(I)-F_{i}^{--}(I)=(L) \int_{I}\left\{f_{i}^{+}-f_{i}^{-}\right\} d \nu=(H) \int_{X_{i} \cap I} f d \nu,
$$

since, once again, $f_{i}^{+}$and $f_{i}^{-}$vanish almost everywhere outside $X_{i}$. Then, by Lemma 7 and Theorem 9, we see that for each interval $I$,

$$
F(I)=\lim _{i \rightarrow \infty} F_{i}(I)=\lim _{i \rightarrow \infty}(H) \int_{X_{i} \cap I} f d \nu=(H) \int_{I} f d \nu .
$$

Since $F$ is finitely additive, for any elementary subset $E_{0}$ of $E$, we have $F\left(E_{0}\right)=(H) \int_{E_{0}} f d \nu$ as desired. The proof of the uniqueness of such a function $f$ follows from the known fact that, if the primitives of two Henstock integrable functions agree on every elementary subset of an elementary set $E$, then the two functions agree almost everywhere in $E$. (See [1, Theorem 5.7].) This completes the proof.

We remark that in view of Theorem 9, the derived sequence of the primitive $F$ of a Henstock integrable function satisfies the basic condition with $F$. In other words, that its derived sequence satisfying the basic condition with itself is a property of the primitive and is therefore not an additional condition imposed on $F$ in Theorem 10. The same applies to the strong $A C G_{\Delta}$-condition as seen in the remark after Definition 5 .

We shall call the function $f$ obtained in Theorem 10 the Radon-Nikodým derivative of $F$ on $E$ and we say that $F$ is Radon-Nikodým differentiable on $E$. We shall also use the notation

$$
f=D_{R N} F .
$$

Note that since a Radon-Nikodým differentiable function is the primitive of a Henstock integrable function, it is a finitely additive and strongly $A C G_{\Delta}$ function on $E$. 
With Theorem 10, we can now have a complete characterization of the primitive of a Henstock integrable function on $n$-dimensional Euclidean space.

Theorem 11. An interval function $F$ is the primitive of a Henstock integrable function $f$ on $E$ if and only if $F$ is a finitely additive strongly $A C G_{\Delta}$ function on $E$ such that the derived sequence of $F$ satisfies the basic condition with $F$.

As we can see, Theorem 11 serves as a descriptive definition of the Henstock integral on $n$-dimensional Euclidean space.

Given a point function $G$, we may define a corresponding additive interval function $F$, called the associated interval function and conversely. (See [3, page 128].) We say that a point function $G$ with associated interval function $F$ is differentiable on $E$ with derivative $G^{\prime}$ if for each $x \in E$, for every $\varepsilon>0$, there is a positive number $\delta_{x}$ such that

$$
\left|F(I)-G^{\prime}(x) \nu(I)\right|<\varepsilon \nu(I)
$$

whenever $I \subset S\left(x, \delta_{x}\right)$ and where $x$ is a vertex of $I$. With this concept, we can prove the following corollary as a simple consequence of Theorem 10.

Corollary 12. Let $G$ be a real-valued point function defined on an elementary set $E \subset \mathbb{R}^{n}$ and let $F$ be the associated interval function. Suppose $G$ is differentiable on $E$ with derivative $G^{\prime}$. Then $G^{\prime}=D_{R N} F$ almost everywhere in $E$.

Proof. For each $x \in E$ and for every $\varepsilon>0$, let $\delta_{x}>0$ be such that (7) holds for all intervals $I \subset S\left(x, \delta_{x}\right)$ where $x$ is a vertex of $I$. Letting $\delta(x)=\delta_{x}$ for each $x$ in $E$, we obtain

$$
\text { (D) } \sum\left|G^{\prime}(x) \nu(I)-F(I)\right|<\varepsilon \nu(E)
$$

for each $\delta$-fine division $D$ of $E$. Then $G^{\prime}$ is Henstock integrable by applying the Henstock Lemma. In fact, for each elementary subset $E_{0}$ of $E$, we have (H) $\int_{E_{0}} G^{\prime} d \nu=F\left(E_{0}\right)$. Then the result follows from the uniqueness of the Radon-Nikodým derivative.

We shall end our discussion in this note by deducing from Theorem 10 two known results for Henstock integrals on the real line. In what follows the function $F$ is a point function.

Corollary 13. (The Fundamental Theorem of Calculus). Let $[a, b]$ be an interval on the real line. If $F$ is a point function differentiable on $[a, b]$ with derivative $F^{\prime}$, then $F^{\prime}$ is Henstock integrable on $[a, b]$ and 


$$
\text { (H) } \int_{a}^{b} F^{\prime}=F(b)-F(a) .
$$

Proof. The result follows by applying Theorem 10 for the case when $n=1$ and Corollary 12.

Corollary 14. Let $[a, b]$ be an interval on the real line. A function $f$ defined on $[a, b]$ is Henstock integrable on $[a, b]$ with primitive $F$ if and only if $F$ is an $A C G^{*}$ function such that the derivative $F^{\prime}$ exists and $F^{\prime}(x)=f(x)$ almost everywhere in $[a, b]$.

Here $F$ is $A C G^{*}$ if $[a, b]$ is the union of closed sets $X_{1}, X_{2}, \cdots$ such that, for each $i$, the following condition holds : for every $\varepsilon>0$, there exists $\eta>0$ such that, for any partial partition $P=\{[u, v]\}$ of $[a, b]$ with $u$ or $v$ in $X_{i}$ satisfying the condition $(P) \sum|v-u|<\eta$, we have $\sum|F(v)-F(u)|<\varepsilon$. (See [3] or [6].) The definition of $A C G_{\Delta}$ can be viewed as a generalization of the definition of $A C G^{*}$ to higher dimensions. As it is shown in Corollary 14, the $(L)$-condition and the basic condition which we impose on the function $F$ of intervals in $n$-dimensional Euclidean space is superfluous when $n=1$ and if $F$ is $A C G^{*}$.

\section{References}

[1] R. Henstock, Lectures on the Theory of Integration, World Scientific, 1988.

[2] E. Hewitt and K. Stromberg, Real and Abstract Analysis, Springer-Verlag, New York, Heidelberg, Berlin, 1969.

[3] P. Y. Lee, Lanzhou Lectures on Henstock Integration, World Scientific, 1989.

[4] P. Y. Lee, Measurability and the Henstock Integral, Proc. Internat. Math. Conf. 94, Kaohsiung, World Scientific, 1995, 99-106.

[5] P. Y. Lee and T. S. Chew, Integration of Highly Oscillatory Functions in the Plane, Proc. Asian Math. Conf. 1990, Hong Kong, World Scientific, 1992, 277-279.

[6] S. Saks, Theory of the Integral, 2nd ed. revised, New York, 1937. 